\title{
ON THE PYTHAGOREAN TRIPLES' EQUATIONS AND THE PERFECT CUBOID PROBLEM.
}

\author{
Alex Nguhi*,1, Cleophas Kweyu ${ }^{1}$
}

\begin{abstract}
The perfect cuboid problem is a Pythagorean problem in nature. This paper gives several propositions regarding Pythagorean relationships and a discussion is made on the perfect cuboid problem. Among the propositions is that the cuboid problem is a divisibility by 3 problem. Violation of the divisibility means that a perfect cuboid doesn't exist for a given integer.

Another consequence of divisibility by 3 and other theorems is that the fastest and most accurate algorithm for generating a random prime number $p$ is,

$$
p=\frac{(5 \times \beta)^{2}+1}{2}
$$

where $\beta=2 k+1, k \in \mathbf{N}$.
\end{abstract}

\section{INTRODUCTION}

Over two thousand years ago, Pythagoras put forward his theorem describing the relationship between two orthogonal sides of a triangle $a$ and $\mathrm{b}$ and the hypotenuse, $c$. By the account of math historians, the study of right-angled triangles existed long before Pythagoras and the tradition has continued ever since, [2]. The perfect cuboid problem studied by Swiss mathematician, Leonard Euler, extends all the possible ideas of Pythagorean relationships into one, $[5,7]$.

\section{PYTRHAGORAS THEOREM AND SQUARES}

The Pythagorean theorem is a geometrical relationship but is manifested in Number Theory [3]. First let us describe squares of numbers in the most elementary way.

\section{Proposition 1.1}

The square of any number can be expressed as the sum of all odd numbers up to to a point. This can be observed by adding the series $1,3,5,7,9,11,13$, $15,17,19, \ldots$ which gives the below output : $1,4,9,16,25,36,49,64,81,100 \ldots$ Fibonacci defined this sum and consequently there are many primitive triples on this line,[8]. It can compactly be written as :

\footnotetext{
${ }^{1}$ Moi University, BOX 3900-30100, Eldoret.

*alexnguhi@gmail.com, kweyuc@gmail.com.
} 


$$
\begin{gathered}
a^{2}=\sum_{k=0}^{a-1}(2 k+1) \\
a^{2}=1+3+5+\ldots 2 a-1
\end{gathered}
$$

This can easily be verified to be true for any number.

Illustration:

$5^{2}=1+3+5+7+(2 \times 5-1)=25$

Let $a, b, c$ be the three sides of a right triangle satisfying the integer condition this means $a \neq b$ or $b \neq c$ because $a^{2}+a^{2}=2 \times a^{2}$ then the other side $c=\sqrt{2} \times a$

So our relation from now on is as follows, $a<b<c$

The Pythagoras theorem maybe dissected below as sums,

$$
\begin{gathered}
a^{2}+b^{2}=c^{2} \\
\sum_{k=0}^{a-1}(2 k+1)+\sum_{k=0}^{b-1}(2 k+1)=\sum_{k=0}^{c-1}(2 k+1)
\end{gathered}
$$

Notice that one sum, $a$ is being appended to the other, integer solutions exist where the numbers $a, b$ maintain their continuous set of odd numbers without any omission. This only happens for odd $a$.

\section{PYTHAGOREAN TRIPLES OF ODD $a$, for $a<b<c$}

\section{Proposition 1.2}

For arbitrary sum of two integer squares such that for $a, b ; a<b$ then $a, b$ form a unique Pythagorean triple if and only if $a^{2}$ is the next odd number after $2 b-1$ belonging to the sum $\sum_{k=0}^{b-1}(2 k+1)$, in other words $a^{2}=(2 b-1)+2=2 b+1$.

So :

$$
\begin{gathered}
b^{2}+a^{2}=c^{2} \\
b^{2}+2 b+1=c^{2}=(b+1)^{2}
\end{gathered}
$$

An example for instance

$$
\begin{aligned}
3^{2}+4^{2} & =(1+3+5)+(1+3+5+7) \\
= & (1+3+5+7)+9=25
\end{aligned}
$$

Let $\theta$ denote the set of all odd numbers greater than one, if we let $a \in \theta$ then a has unique solutions for the entire space.

This has been proven repeatedly in many forms by many scholars, $[2,3,7]$ but generally,

$$
c=\frac{a^{2}+1}{2}, a \in \theta, \theta=3,5,7,9 \ldots
$$


All numbers produced by $\theta$ are commonly referred to as primitive along with others produced by an even $a$. In this exploration they will be called 'perfect' because they have some nice properties.

\section{Definition 1.1}

Primitives of odd a are 'perfect' if and only if they maintain the relation $a<b<c$. Another special definition of perfectness will be given later for even $a$.

Properties of perfect triples of $a \in \theta$ are as follows :

1.At-least one the elements is divisible by 3 or 5 or both, although this is common for any triple.

2.At-least one of the sides is prime for odd $a$. This property is not necessary because some other conditions need be satisfied.

3.The elements maintain the condition $a<b<c$. This is a strong property and must be preserved.

\section{PYTHAGOREAN TRIPLES OF EVEN $a$, for $a<b<c$}

Primitive Pythagorean triples also exist for even $a$, satisfying $a<b<c$ and by primitive they aren't scalars of the previous case where $a$ is odd i.e $2 \mathrm{k}(\mathrm{a}, \mathrm{b}, \mathrm{c})$ is also a non primitive-Pythagorean triple.

The mathematics of such numbers is not as straightforward as the 'Appending method' of odd triples done earlier on. The beast can however be tamed and consistent equations derived.

\section{Theorem 1.1}

For any perfect Pythagorean triples $a<b<c$ such that $a$ is even, $b$ and $c$ must satisfy the equation $b+c=2 r^{2}$ for $r \in \theta^{*}$ given that:

$$
c-b=2 k^{2 n}
$$

Generally:

$$
\begin{gathered}
a=r \times 2^{m} \\
b=r^{2}-\epsilon \\
c=r^{2}+\epsilon
\end{gathered}
$$

where $\theta^{*}$ denotes the set of integers greater than 3 . 


\section{Case 1 - Odd $\theta^{*}$}

There exists a triple in the neighborhood of $25,49,81,121,169 \ldots$. The value of $\mathrm{k}=2$.

Equations describing triples:

$$
\begin{gathered}
c=b+2^{2 n+1} \\
=r^{2}+\epsilon=b+2^{2 n+1}
\end{gathered}
$$

therefore

$$
b=r^{2}+\epsilon-2^{2 n+1}
$$

and $\epsilon$ can be defined by $\epsilon=\frac{2^{2 n+1}}{2}=2^{2 n}$

So that we have a Diophantine equation giving solutions of $b$ is as follows :

$$
b=r^{2}+2^{2 n}-2^{2 n+1}
$$

Examples :

Consider the Pythagorean Triple $(44,117,125)$ It follows that,

$$
\begin{aligned}
& a=11 \times 2^{2} \\
& b=11^{2}-4 \\
& c=11^{2}+4
\end{aligned}
$$

For another $(104,153,185)$

$$
\begin{aligned}
& a=13 \times 2^{3} \\
& b=13^{2}-16 \\
& c=13^{2}+16
\end{aligned}
$$

The third triple $(120,209,241)$

$$
\begin{aligned}
& a=15 \times 2^{3} \\
& b=15^{2}-16 \\
& c=15^{2}+16
\end{aligned}
$$

These are not the only solutions, in-fact we can give a solution that shifts the sides as an example, 


$$
\begin{aligned}
& b=15^{2}-64=161 \\
& c=15^{2}+64=289 \\
& 289^{2}-161^{2}=240^{2}
\end{aligned}
$$

The elements still observe $c-b=2^{2 n+1}$ rule given above , $289-161=2^{7}$ So that the triple is $(161,240,289)$, in this case the even number is sandwiched between two odd numbers. One important discovery is that for any sufficiently large odd number $r$ we can find many Pythagorean triples.

\section{Case 2 - Even $\theta^{*}$}

Now let's consider when $\mathrm{r}$ is is even so that primitive triples lie in the neighborhood of $16,36,64,100, \ldots$, i.e. the set of even square numbers.

For instance $8,15,17$ and 20,99,101 and may be generalized by :

$$
\begin{gathered}
b+c=2 r^{2} \\
b=r^{2}-\epsilon \\
c=r^{2}+\epsilon
\end{gathered}
$$

but the following change : $c-b=2 \times 3^{n}, \epsilon=3^{n}$, so that $b$ and $c$ are the odd numbers that determine $a$.

From theorem $1.1 c-b=2 k^{2 n}$, Here $\mathrm{k}=3$

Similar examples:

$48,55,73$

$$
\begin{gathered}
55+73=128=2 \times 8^{2} \\
55=64-9,73=64+9
\end{gathered}
$$

$60,91,109$

$$
\begin{gathered}
109+91=200=2 \times 10^{2} \\
91=100-9,109=100+9
\end{gathered}
$$

$8,15,17$ given above has $\epsilon=3^{0}=1$,

Some of the primitives here also satisfy perfectness by $a<b<c$ where $a$ is even

\section{Consequences of Theorem}


If the $(a<b<c)$ rule is violated then it affects the distribution of primes so that for some primitive triples there's no prime number present. The reason for this will be explored in further detail through divisibility by 3 and 5 .

\section{Types of Primitive Configurations and their Solutions}

All the triples $a<b<c$ for $(\mathrm{a}, \mathrm{b}, \mathrm{c})$

1.Archetype $1-a$ is odd, $b$ is even and $c$ is odd. given by $c=\frac{a^{2}+1}{2}$

Without using Pythagorean Theorem we can generalize it into:

$$
b+c=a^{2}
$$

2.Archetype $2-a$ is even,$b$ is odd and $c$ is odd. These are defined by Theorem 1.1 for perfect triples.

Summarized by the formula:

$$
b+c=2 r^{2}
$$

3.Archetype 3- $a$ is odd, $b$ is even and $c$ is odd. This one follows theorem 1.1 but breaks the rule $a<b<c$. In the previous section it was suggested that breaking the perfectness condition also coincidentally breaks the prime condition for some triples.

Given by:

$$
\begin{gathered}
\hat{a}+\hat{c}=2 r^{2} \\
\hat{b}+\hat{c}=\hat{r}^{2}
\end{gathered}
$$

and that $\hat{r} \neq r$.

Let's define some few other theorems that will define prime distribution.

\section{Theorem 1.2}

For any Pythagorean triples, at least one element is divisible by 5.

\section{Proof:}

The proof is done through terminal digits for archetype 1. Archetypes 2 and 3 are harder to prove but the principle remains nonetheless if moduli and quadratic expansions are applied.Archetype 1 is of interest because of prime numbers.

From the previous section, $c=\frac{a^{2}+1}{2}$ with the choice $a \in \theta, \theta=3,5,7,9 \ldots$

All odd numbers have 1,3,7,5,9 as their terminal digits, hence, any odd number's square ends in the terminal digits $1,9,5,9,1$ 
The operation requires an addition of 1 then division by 2 , which results in the terminal digits : $\{1,5,3,5,1\}$ and the Corresponding values of $b$ being $\{0,4$, $2,4,0\}$ with $c=b+1$.

Arranging the three expressions of terminal digits looks as follows :

$$
\begin{gathered}
\text { a - }\{1,3,5,7,9\} \\
b-\{0,4,2,4,0\} \\
\text { c - }\{1,5,3,5,1\}
\end{gathered}
$$

The theorem can easily be proved by checking the vertical combinations and because all of them contain either 0 or 5 which means the Theorem is true for archetype 1.

Therefore there are infinitely many solutions divisible by 5 .

\section{Theorem 1.3}

For any given Pythagorean triples then one integer is divisible by 3 .

\section{Proof and Discussion}

Like theorem 1.2, this can easily be proven for Archetype 1.

From a short numerical analysis then, we can derive a several equations describing all unique solutions for $a, b$ and $c$ and from them derive some inference

$$
\begin{gathered}
a=3,5,7,9,11,13 \ldots \\
b=4,12,24,40,60,84 \ldots \\
c=5,13,25,41,61,85 \ldots
\end{gathered}
$$

All the sets can be redefined by the following equations :

$$
\begin{gathered}
a=1+2 k, \text { with } k=1,2,3 \ldots \\
b=\sum_{k=0}^{n} 4 k+4, k \in N \\
c=\sum_{k=0}^{n} 4 k+5, k \in N
\end{gathered}
$$

Our closed Pythagorean equations tell us that the sets $\mathrm{a}$ and $\mathrm{b}$ alternate divisibility by 3 . If $a$ is divisible by three then $b$ is not and the reverse is also true. Hence the partial visual proof of the theorem. Divisibility by $3,4,5$ has been explored in the past by various mathematicians like Dickson in the groundbreaking work in Diophantine analysis, [1]. 
In this case I'll apply Mathematical logic to derive a more concrete method to prove it's true for archetype 1 . The trend is a follows for $a$ and $b$ :

$$
\begin{gathered}
3 \times 1+1=4 \\
5 \times 2+2=12 \\
7 \times 3+3=24 \\
9 \times 4+4=40 \\
11 \times 5+5=60 \\
13 \times 6+6=84 \\
15 \times 7+7=112 \\
\ldots \ldots \ldots \\
2 p+1 \times p+p=m
\end{gathered}
$$

For , $m \in \mathbf{N}$ and $m=2 p^{2}+2 p$

If we accept that this is another way to express $a, b$ then we can deduce the following for an odd number $k$ in the series that with $2 p+1=k$ :

case 1

The odd number $k$ and $\frac{k-1}{2}$ have the same modulus and multiplying them shifts the modulus from 1 to 2 and vice versa, so adding $\frac{k-1}{2}$ now means that the modulus is 0 .

case 2 :

Where $\frac{k-1}{2}$ is divisible by 3 is trivial because the modulus will be 0 automatically. Therefore by mathematical induction, the assertion is true.

Another argument may be made for archetype 3 and 2 . But let's establish that:

1. All square numbers, $r^{2}, r \in \theta^{*}$ not divisible by 3 are of mod 1 when divided by 3 . i.e $16,25,49,64,100,169 \ldots$ due to a situation similar to previous one for odd numbers of shifting moduli

2. Even numbers of mod 1 maintain their modulus after squaring. Even numbers of mod 2 behave like odd numbers and shift their moduli.

If that be the case then since the elements are equidistant from the square number by $2^{n}$ it means that at-least $r^{2}-2^{n}$ is divisible by 3 because the powers of 2 also shift moduli - Consider $1,4,8,16,32,64,128 \ldots$.

\section{Example :}

For 25 , the radius in the triple $(20,21,29)$ is of mod 1 when divided by 3,4 is mod of 1 , therefore $25-4$ is divisible by 3 , hence suggesting that at least one number $a, b$ is divisible by 3 . 
Another example using the triple $(161,240,289)$ in the radius 225 , which is divisible by 3 hence mod 0 . The sides $a, c$ lie in the zone $225 \pm 64$. 64 is of mod 1 when divided by 3 so, c must be divisible by 3 because $c^{2}=289^{2}-169^{2}$

For the Archetype 2 where $b$ and $c$ lie on the even $r^{2}$ with $\epsilon=9$, the solution is trivial because $\epsilon$ doesn't affect their moduli at all. This means that $c^{2}-b^{2}$ is of modulus 0 and happens to be $a^{2}$ suggesting a deep harmony in divisibility by 3 .

\section{Corollary 1.2}

For any primitive Pythagorean triple the integer c cannot be divisible by 3. Divisibility by three is only reserved for spots a and $b$ only.

Two prime generating algorithms will rely on divisibility and create accurate prime number sieves .

\section{Algorithm 1}

The most efficient algorithm for obtaining prime a number, $p$ is given by

$$
p=\frac{(5 \times \beta)^{2}+1}{2}
$$

where $\beta$ is an odd number

There are infinitely many primes of this form and they all lie on the solutions of the above Diophantine equation spread over the Odd space of numbers.

First 50 odd $\beta$ solutions , all primes: 13, 113, 313,613, 1013, 2113, 3613, 4513, 10513, 13613, 15313, 19013, 2101

This sieve is analogous to Mersenne Primes, [4]. Not all numbers that satisfies $2^{n}-1, n \in \mathbf{N}$ are prime numbers and so is the case of the above equation, For instance when $\beta=21$ then the number 5513 is not prime because it's divisible by 37,149

The above equation however has more dense solutions.

\section{Algorithm 2}

This algorithm combines theorem 1.1,1.2,1.3 with archetypes 2 and 3. If we agree that for any Pythagorean triple there exist two numbers such that one is divisible by 5 and another by 3 , then:

1. $c$ is a prime number if and only if $b$ is divisible 5 and $a$ is divisible by 3 .

e.g $48,55,73$

2. $c$ is a prime number if and only if $b$ is divisible 3 and $a$ is divisible by 5 . 
e.g $65,72,97$

3. $c$ is prime if $b$ is divisible by both 3 and 5 e.g $(28,45,53)$ and $(8,15,17)$

Since archetype 1 and archetype 3 are similar, then this algorithm can be applied for either case.

\section{Corollary 1.1}

If either $a$ or $b$ is not divisible by 5 then $c$ cannot be a prime number because it must be divisible by 5 so as to satisfy theorem 1.2

For example $(33,56,65)$

\section{The Pythagorean Triples Space and Approximations}

\section{Archetype 1}

Because of abstraction let's start with an example for clarity before a general form. Consider $k_{1}=17, k_{2}=3, k_{3}=5, a=k_{1} k_{2} k_{3}$ has the following dimensions produced by the formula $c=\frac{a^{2}+1}{2}, a \in \theta, \theta=3,5,7,9 \ldots$

$(255,32512,32513)$

$$
\begin{gathered}
15(17,144,145)=255,2160,2175 \\
17(15,112,113)=(255,1904,1921) \\
3(85,3612,3613)=(255,10836,10839)
\end{gathered}
$$

There exists a unique scaling between the Principal hypotenuse 32513 and all the other combinations' hypotenuses. From this point the largest hypotenuses will be called 'Principal hypotenuse'.

$$
\begin{aligned}
& \text { Observe } \\
& \begin{array}{l}
\frac{32513}{15} \approx \frac{2160+2175}{2} \\
\text { So }
\end{array} \\
& 2167.5333 . . \approx 2167.5 \\
& \frac{32513}{17} \approx \frac{1904+1921}{2} \\
& \text { 1912.529.. } \approx 1912.5 \\
& \frac{32513}{3} \approx \frac{10836+10839}{2} \\
& 10837.66 . . \approx 10837.5
\end{aligned}
$$


From the above example a scaling is visible, in-fact the approximation can be turned into an equality if we defined an exact error rate function.

\section{Theorem 1.3}

Let $p$ be a principal hypotenuse for an odd number $a$ such that $a=k_{1} k_{2} k_{3} \ldots k_{n}$ and $x_{i}, y_{i}$ are other two sides produced by the various combinations.

Then,

$$
\begin{gathered}
\frac{p}{k_{i}}-\epsilon=\frac{x_{i}+y_{i}}{2} \\
\text { where } \epsilon=\left(\frac{p}{k_{i}}-\frac{x_{i}+y_{i}}{2}\right)^{-1}
\end{gathered}
$$

A further deduction can be made that $(\epsilon)^{-1}=2 k_{i}$. So our generalized Scaling Theorem becomes :

$$
\frac{p}{k_{i}}-\frac{1}{2 k i}=\frac{x_{i}+y_{i}}{2}
$$

Example:

Combinations belonging to the principal 32513:

$(255,32512,32513)$

$3(85,3612,3613)=(255,10836,10839)$

Applying the scaling theorem gives:

$$
\left(\frac{32513}{3}-\frac{1}{2 \times 3}=\frac{10836+10839}{2}\right.
$$

\section{Mean Values Involving Archetype 1 and 2}

Consider two Pythagorean triples sharing 44 as the value of $a$ :

$$
\begin{gathered}
4(11,60,61)=(44,240,244) \\
(44,117,125)=(44,117,125)
\end{gathered}
$$

Notice that:

$$
(125+117)=\frac{240+244}{2}=242
$$

Example 2, $a=104=8 \times 13$ 


$$
\begin{gathered}
8(13,84,85)=(104,672,680) \\
(104,153,185) \\
(153+185)=338 \text { and } \frac{672+680}{2}=676=2 \times 338
\end{gathered}
$$

\section{Proposition 1.3}

If $a$, an even number contains solutions for both archetype 1 and 2, then the sum of their $b$ and $c$ sides, $S_{i}$ satisfies,

$$
S_{2}=\frac{S_{1}}{2 k}
$$

, where $\mathrm{k}$ is an integer. This is directly derived from the above example.

Another argument may be made for Archetype 3 and 1 since the radii are preserved and hence have similar properties.

$$
\hat{S}_{2}=\frac{S_{1}}{k}
$$

In this case $a$ and $\hat{a}$ are odd numbers and are equal.

Generally the idea behind this is to have the smallest side being shared by several triples but $b$ 's and c's aren't equal at all.

\section{THE PERFECT CUBOID PROBLEM}

Consider the cuboid below :

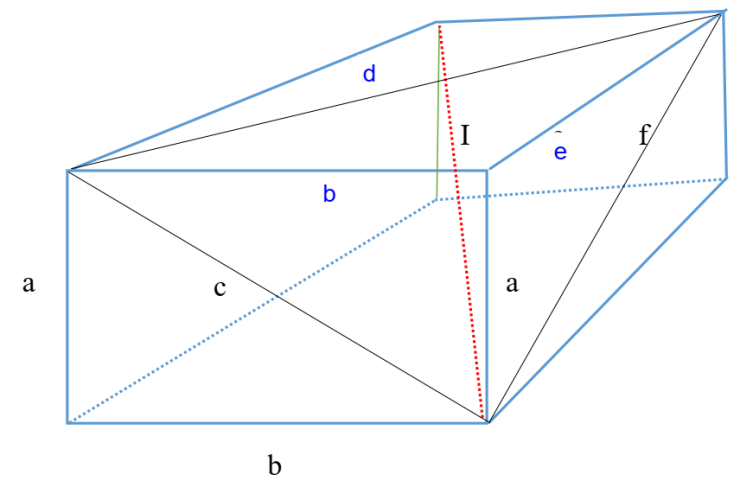

Figure 1: cuboid 
A perfect cuboid is defined as one which all the dimensions - triangles enclosed within it are of integer values. Until recently the study was restricted to real numbers but it's possible to have a cuboid with atleast one side being in the complex space.

A hypothetical Euler brick can easily be traced out by the black diagonals c,d,f. It must have integer elements for this triangle but the rest of the cuboid doesn't have to be perfect.

By perfect it doesn't imply 'perfect primitive' even though the problem could be modeled around that. It means that all the dimensions of the cuboid have integer values including the interior diagonal $I$ ( The interior diagonal is denoted by the red dotted line.) More info can be found in $[5,6]$

For any Cuboid, only the diagonals and rectangles have equal size and consequently three unique right-angled triangles share the shortest side $a$ and if the smallest triangle is $(a, b, c)$, we have an additional triangle with $b$ as the shortest side. The relationship of the dimensions is as follows:

$$
a<b<c<e<f<d<I
$$

When the cuboid is translated into $1-D$ space one is able to obtain 4 triangles. The problem can then be formulated basing on these triangles rather than their actual solutions.For the analysis to be consistent, the element of interest must be smallest side that also shares a side with the interior diagonal $I$.

\section{Reformulation of the Perfect Cuboid Problem.}

The problem may be generalized into: For right angled triangles $(a, e, f)$, $(a, b, c),(a, d, I)$, can the sandwiched elements $e, b$ and $d$ form a Pythagorean triple of pure integers, $(b, e, d)$ ?

\section{Important Deductions:}

If we agree that atleast one of the sides of a Pythagorean triple is divisible by 3 and that all the sides are divisible by 3 if and only if a scaling is done by $\delta=3$.

So, now suppose $a$ is divisible by 3 and scaled by $\delta \neq 3$ then this suggests that $b, e$ and $d$ might not be be divisible by 3 and thus making the perfect

cuboid non-existent at that point of $a$ because at least one integer needs to be divisible by 3 .

Now consider a case where $a$ is not divisible by 5 or 3 . We can say with absolute certainty that another casualty exists because $b, e$ and $d$ must all be divisible by 3 , making the perfect cuboid in-existent. 
For $d$, if $d$ is divisible by 3 then, $b$ and $e$ must also be divisible by 3 which suggests that $a$ is scaled by 3 which also means nothing because it can be scaled out for a given triple(primitives are unique and their scaled triples too). This is because the diagonal $d$ is never divisible by 3 from previous propositions. However mathematical logic is not as satisfactory as equations, so we could be stuck at a deadlock easily.

However, an even more important question remains, can the mean values generated by previous propositions above allow the existence of a perfect cuboid ?

\section{Archetypal Equations, Mean Values and the Cuboid Problem}

Consider the previous equations, $b+c=a^{2}, b+c=2 r^{2}, \hat{a}+\hat{c}=2 r^{2}, \hat{b}+\hat{c}=\hat{r}^{2}$ . To attempt to solve the cuboid problem we can start by choosing atleast one archetype and assign it to one side.

For instance, $(a, e, f)$ will be of archetype 1, therefore

$$
\begin{gathered}
e+f=a^{2} \\
a=\sqrt{e+f}
\end{gathered}
$$

So now we can replace $a$ so that the triangles are :

$(\sqrt{e+f}, e, f)$

$(\sqrt{e+f}, b, c)$

$(\sqrt{e+f}, d, I)$

The next step involves choosing another suitable side and archetype, so we'll go for archetype 2 to avoid large scaling so that $b<e$,

Then

$$
\begin{aligned}
& b+c=2 r^{2} \\
& b=2 r^{2}-c
\end{aligned}
$$

The the third triangle $(a, d, I)$ can be set to be of archetype 1, then we can apply the previous approximations such that:

$d+I=2 k(b+c)$

Substituting for $b$,

$$
d+I=2 k\left(2 r^{2}-c+c\right)=4 k r^{2}
$$

So if a combination that of triples satisfies $(\sqrt{e+f}, e, f)$ and $d+I=4 k r^{2}$, ideally it could be a perfect cuboid if $b=2 r^{2}-c$.

Notice that side $\boldsymbol{a}$ must be even to satisfy Archetype 2, giving a glaring false assumption for Archetype 1 . Normally, $a, e, f$ can't be primitive and at the same time produce an archetype 2 for $(a, b, c)$. 
To make the Diophantine equation work either choose the first triple to be of archetype 3 or do a rescaling.

The false assumption was that $a, e, f$ is scaled by 1 ,now it can rescaled by $\delta$, this ensures that archetype 2 has the smallest side divisible by 2 as follows below.

$$
\begin{gathered}
\delta(a, e, f) \\
(\delta \sqrt{e+f}), \delta e, \delta f)
\end{gathered}
$$

If $a, e, f$ is not primitive then it violates $e+f=a^{2}$ so, it'll have to be scaled out before the implicit addition. However, one upside of these equations is that they can be used as a litmus test for primitive Pythagorean triples.

$\delta$ will then be distributed to the other sides depending on primitivity of the second triple(of Archetype 2). If it's primitive, $c, b$ are unique and not factorizable by $\delta$ for the triangle $(\delta a, b, c)$. If so,

$$
b+c=2 r^{2}
$$

$(\delta a, d, I)$ can be of archetype 1 or 3 , previously it was of archetype 1 , so $d$ and $I$ are uniquely determined by the prime factorization of $a$ and not entirely on $\delta$. This yields a similar equation

$$
d+I=2 k\left(2 r^{2}-c+c\right)=4 k r^{2}
$$

So are there any integers $d, I, k, r$ that satisfies the equation, $d+I=4 k r^{2}$ ?

If $(a, d, I)$ is of archetype 3 and non-primitive, then we can introduce another variable $z$ to take care of the scaling

$$
\delta a+I=z \hat{r}^{2}
$$

Notice that the $\delta$ scaling can be taken care of by $z$. So that now $z$ will be $\hat{z}$ such that $z<\hat{z}$, it'll just increase in value without any effect on the system, $\delta a$ can simply be substituted with $a$. This is because the initial triangles retain their ratios. This is a neat caveat because the more the free and redundant variables, the more the calculation complexity.

$$
\begin{aligned}
& a+I=\hat{z} \hat{r}^{2} \\
& a=\hat{z} \hat{r}^{2}-I
\end{aligned}
$$

Since $b, c$ are unique for $(\delta a, b, c)$ triple then the same can be done giving,

$$
a^{2}=c^{2}-b^{2}
$$


And $a=\sqrt{c^{2}-b^{2}}$, then

$$
\begin{gathered}
a=\hat{z} \hat{r}^{2}-I=\sqrt{c^{2}-b^{2}} \\
\hat{z} \hat{r}^{2}-I=\sqrt{c^{2}-\left(2 r^{2}-c\right)^{2}} \\
\hat{z} \hat{r}^{2}-I=\sqrt{-4 r^{4}+4 c r^{2}} \\
\hat{z} \hat{r}^{2}-I=2 r\left(\sqrt{-r^{2}+c}\right)
\end{gathered}
$$

The triple can be turned to primitive by setting $z=1$. This also gives possibilities of complex numbers.

The challenge with this is that it requires 5 parameters which are more than the previous case of Archetype 1 for $(a, d, I)$. However this implies that all triangles will be taken care of by just one equation

The above Diophantine equations are to be observed by any perfect cuboid if it exists for the respective archetypes. Nonetheless other equations can be derived depending on the choice of the archetypes.

\section{CONCLUSIONS}

Perfect primitives give a helpful numerical analysis of prime numbers. If two triples share the same value for $a$, such that $a<b<c$ then there exist a relationship between their respective $b$ 's and $c$ 's . This is the underlying principle of the perfect cuboid.

\section{REFERENCES}

1. Dickson, L. E. (2013). History of the theory of numbers: Diophantine Analysis (Vol. 2). Courier Corporation, 171.

2. Maor, E. (2019). The Pythagorean theorem: a 4,000-year history. Princeton University Press, $5-6$.

3. Hartshorne, R. (2013). Geometry: Euclid and beyond. Springer Science \& Business Media, 42 .

4. Robinson, R.M.(1954) . Mersenne and Fermat numbers. Proceedings of the American Mathematical Society 5(5), 842-846.

5. R. Guy, Unsolved Problems in Number Theory, 3Eds., New York: Springer, 2004. Fitzpatrick, R. (2007). Euclid's elements of geometry. Euclidis Elementa,275.

6. Weisstein, E.W(2002).Cuboid.https://mathworld.wolfram.com

7. Butler, Bill. "Durango Bill's The 'Integer Brick' Problem(The Euler Brick Problem". https://www.durangobill.com/IntegerBrick.html

8. Sigler,L.E(ed)(2014).Leonardo Pisano(Fibonacci). The Book of Squares. Elsevier 